\title{
Behavioral Health Risk and Resilience Among International Students in the United States: A Study of Sociodemographic Differences
}

\author{
Youn Kyoung Kimª , Arati Malekub ${ }^{\mathrm{b}}$, Catherine Lemieu ${ }^{\mathrm{a}}$, Xi Du ${ }^{\mathrm{a}}$ and Zibei \\ Chen $^{\text {c }}$
}

\begin{abstract}
Using a resilience framework, the current cross-sectional study examined indicators of behavioral health risk and resilience among U.S. international students $(\mathrm{N}=322)$ across key sociodemographic characteristics. A multimethod approach was used to collect data with both an online platform and paper-based survey instrument. Results showed that higher levels of acculturative stress were reported by older students, females, undergraduates, students who lived with their families, and those who had resided in the US longer than 2 years. Findings underscore the importance of culturally relevant screening and prevention strategies that target resilience and other protective factors to reduce health risk and encourage well-being and academic success among international students
\end{abstract}

Keywords: acculturative stress, alcohol use, anxiety, depression, international students, resilience

\section{Introduction}

International students have an ever-increasing presence in colleges and universities worldwide. The US has, by far, the largest number of international students, a population that has consistently increased in recent years (Institute of International Education [IIE], 2016). International students enrich the educational landscape of U.S. universities and provide unique opportunities for cultural and intellectual exchange, and although the academic and cultural experiences gained in a foreign country are rewarding, international students face both individual (e.g., financial difficulties, language

\footnotetext{
a Louisiana State University.

b Ohio State University.

${ }^{\mathrm{c}}$ University of Michigan.
} 
barriers) and structural challenges (e.g., perceived discrimination, higher academic standards) that can compromise their successful adaptation (Eustace, 2007; Koyama \& Belli, 2011; Sullivan, 2010). Studies show that, as compared to their U.S. counterparts, international students experience disproportionately high rates of behavioral health difficulties (i.e., depression, anxiety, alcohol misuse) that can negatively impact their academic performance and overall well-being (Holguin, 2011; Sa, Seo, Nelson, \& Lohmann, 2013). However, research also has emphasized the importance of culture-based resilience, which can mitigate the impact of acculturative stress and other challenges (Kim \& Kim, 2014; Ungar, 2012). Although U.S. international students represent a diverse group with regard to sociodemographic characteristics, little is known about the behavioral health risk and protective factors among students with varying demographic profiles. Using resilience as the theoretical framework, the present study addresses this gap in the knowledge base by examining behavioral health risks and resilience in a diverse sample of U.S. international students.

\section{Literature Review}

\section{International Students in the United States}

During the 2015-2016 academic year, a total of 1,043,839 international students were enrolled in U.S. colleges and universities, a 7\% increase from the previous academic year (IIE, 2016). According to the Student and Exchange Visitor Program (SEVP, 2014) three states (i.e., California, New York, and Texas) hosted over one third of all U.S. international students $(35 \%)$. The population of international students comprises a demographically diverse group, with SEVP (2014) data showing that the slight, but growing majority is male $(56.3 \%)$. In terms of country of origin, the greatest proportion of U.S. international students is from Asia $(60 \%)$, with smaller proportions from Europe (23\%), Africa (12\%), and other countries (5\%; Organization for Economic Co-operation and Development [OECD], 2013). According to the IIE (2016), approximately one third of Asian students in the US are from China (31\%), with smaller proportions from India and South Korea (at $14 \%$ and $7 \%$, respectively). International students typically migrate to the US to pursue degrees in the science, technology, engineering, and math (STEM) fields, as opposed to the social sciences (IIE, 2016). In terms of specific areas of study, engineering, computer and information science, and business (including management, marketing, and related support services) are the most popular majors chosen by international students.

\section{Acculturation, Acculturative Stress, and Behavioral Health Risks}

As international students adjust to unfamiliar social, cultural, and educational environments, they experience acculturation-a dynamic, complex, and multidimensional process of adaptation (Berry, 2005). This process of acculturation also can adversely impact the well-being of international students due to acculturative stress, the discomfort and difficulties in functioning students experience as they adapt to the language, norms, and values of the host culture (Yang \& Clum, 1995). Acculturative stress is rooted in both intrapersonal (i.e., personality) and environmental factors (Sandhu, \& Asrabadi, 1994), with older international students typically demonstrating slightly higher levels of acculturative stress 
than their younger counterparts (Lau, 2006). Research suggests that religion and spirituality may help some international students cope with the demands of acculturation. For example, Hsu, Krägeloh, Shepherd, and Billington (2009) found that religion/spirituality was positively associated with both psychological and social quality of life among 164 international students in New Zealand. Extending this latter study, Chai, Krägeloh, Shepherd, and Billington (2012) sampled 679 students in New Zealand and found that Asian students were more likely than European students to use religious coping strategies, irrespective of whether they were international or domestic students. Thus, religiosity or spirituality might afford certain minority groups protection when confronted with acculturation stressors.

Low English language proficiency (Poyrazli, Kavanaugh, Baker, \& Al-Timimi, 2004), inadequate social support (Hayes \& Lin, 1994), and perceived discrimination (Ying \& Han, 2006) are well-established factors that exacerbate acculturative stress among international students. For example, using a relatively large sample of diverse U.S. international students ( $\mathrm{N}=359)$, Yeh and Inose (2003) showed that low self-assessed English language proficiency and low levels of social support were independent predictors of psychological distress. Duru and Poyrazli (2007) found that lower levels of English language competency and social connectedness predicted acculturative stress among 229 Turkish students studying in 17 U.S. universities. At one U.S. university, Sherry, Thomas, and Chui (2010) found that due to fear of discrimination because of language difficulties, Chinese students were reluctant to interact with domestic students and subsequently suffered from loneliness and other symptoms of depression and anxiety. Conversely, Poyrazli and Kavanaugh (2006) found that international students who connected socially with students from the host country showed lower levels of stress than those who were more isolated. In a similar vein, Cheung and Yue (2013) found that local connectedness was positively associated with resilience and negatively associated with depressed mood among 215 Mainland Chinese students enrolled in a Hong Kong university.

Acculturative stress is shown to have both proximal and long-term effects on psychological well-being (Ying \& Han, 2006). A substantial corpus of research undertaken with diverse international student samples indicates that acculturative stress is associated with an elevated risk of mental health problems (Constantine, Ogazaki, \& Utsey, 2004; Duru \& Poyrazil, 2007), including depression, anxiety, and misuse of alcohol, tobacco, and illicit drugs (Holguin, 2011; Li, Marbley, Bradley, \& Lan, 2016; Sa et al., 2013; Wei et al., 2007). Hahn (2010) examined interrelationships among stress, coping, cultural orientation, and depression among 648 international students from 74 countries studying at one U.S. institution and found that almost one fourth (22.6\%) met criteria for clinical depression, a rate twice that for domestic students. In a smaller survey of 130 Chinese students at Yale University, Han, Han, Luo, Jacobs, and Jean-Baptiste (2013) found that rates of depression (45\%), and anxiety (29\%) were approximately three times higher than those for domestic students (at $14 \%$ and 9\%, respectively). Although a handful of studies report varying levels of depression and anxiety across gender with some U.S. international student samples (e.g., Khoshlessan \& Das, 2017; Lau, 2006), the research has yielded no differences in rates of depression among international students across 
other characteristics such as length of residency, educational level, religious affiliation, and source of funding (e.g., Han et al., 2013; Wei et al., 2007).

Studies show that alcohol misuse, including binge drinking, is fairly prevalent among U.S. international students (Holguin, 2011) and that higher levels of acculturative stress are linked to stronger motivations to drink alcohol (Sa, 2010). Alcohol misuse can detrimentally impact the overall psychological and physical health of international students, which, in turn, can negatively affect their adaptation and academic performance (Sa, 2010; Yeramaneni \& Sharma, 2009). Research suggests that the fear and culture shock experienced by many international students during the process of acculturation can precipitate the development of negative coping strategies, including the use of alcohol (Kanaparthi, 2009). For example, using a sample of 262 students enrolled in English as a second language programs, Koyama and Belli (2011) found that some students drank alcohol to increase pleasant feelings and to avoid the negative feelings associated with acculturative stress. Based on a qualitative study of 16 international students' health behaviors, Yan and FitzPatrick (2016) suggested that students who socialize with American students by drinking alcohol are vulnerable to developing alcohol-related problems. Similar to the U.S. undergraduate college population (Laudet, Harris, Kimball, Winters, \& Moberg, 2015), co-occurring mental health symptomology is common among international students who misuse alcohol (Kim \& Kim, 2014), with higher levels of depression associated with hazardous use of alcohol (Sa, 2010).

\section{Resilience}

Resilience is the process by which individuals overcome risks and avoid negative outcomes by using internal assets and external resources when confronted with stressful situations (Fergus \& Zimmerman, 2005), and it emerges as a protective factor against certain at-risk behaviors (e.g., binge drinking) across diverse college populations (Hodder et al., 2011; Ungar, 2012). Using a sample of 88 U.S. undergraduate students, Johnson, Dinsmore, and Hof (2011) examined the relationship between resilience and five different levels of alcohol use and found that students with higher levels of resilience reported less alcohol consumption than those with lower levels of resilience. C. Y. Lee and Park (2014) examined the moderating effect of resilience on the relationship between acculturative stress and depression among university students from North Korea $(\mathrm{N}=116)$ and found that resilience buffered the negative impact of acculturative stress on depression.

In the context of students' educational migration, resilience is a critical factor that may enable international students to adapt to environmental change in positive ways (Sa et al., 2013; Yoo et al., 2014). From this perspective, resilience may enhance students' adaptive coping, as well as help them avoid negative outcomes (e.g., mental health symptomology, health risk behaviors) that are detrimental to their overall well-being (Friborg, \& Zimmerman, 2005). However, research undertaken with international students has yielded somewhat mixed results. Using a sample of 290 Chinese international students in Korea, Yoo and colleagues (2014) demonstrated that resilience partially mediated the effect of acculturative stress on negative mental health outcomes; however, resilience was not associated with any key sociodemographic characteristics, such as gender, education level and major. 


\section{Purpose of the Current Study}

Although a substantial body of literature addresses acculturative stress and health-risk behaviors among international students, no study to date has examined a full range of potentially relevant sociodemographic correlates of behavioral health risk and resilience. The extant research has focused only on a limited number of demographic variables (age, gender, ethnicity), and it has yielded mixed findings (e.g., Poyrazli et al., 2004). In terms of ethnicity, studies have focused more so on Asian international students in different geographical areas in the US, and on Chinese international students, in particular (Iwamoto \& Liu, 2010; Lau, 2006; Wei et al., 2007).

Gender emerges as a potentially relevant correlate of both acculturative stress and behavioral health risk and resilience (Koyama \& Belli, 2011; C. Y. Lee \& Park, 2014). Given recent recommendations to incorporate a more gendered perspective into migration research undertaken with international students (Sondhi \& Russell, 2017), additional investigation of the role of gender is warranted. Age, ethnicity, marital status, and English language proficiency also are associated with acculturative stress among international students (Poyrazli et al., 2004). A host of additional educational (educational experience in the US, area of study, academic performance, and sources of funding) and other characteristics (religious affiliation, family status, length of stay in the US) have emerged as relevant correlates of acculturative stress (Chai et al., 2012; Duru \& Poyrazli, 2007; Sullivan, 2010); however, these latter covariates have received little attention in studies specifically assessing behavioral health risk and resilience among international students.

Although acculturative stress can be a chronic stressor among international students who are confronted with unpredictable challenges in a new environment, they generally have the ability to successfully navigate both daily living and academic challenges without engaging in health risk behaviors (Chin, 2016; Ying \& Han, 2006). The role of resilience in relation to sociodemographic characteristics, however, is not well understood. Given the diversity that characterizes U.S. international students, a comprehensive examination of students' sociodemographic profiles will facilitate a better understanding of important sub-group similarities and differences with respect to acculturative stress and behavior health risk and resilience.

\section{Research Method}

\section{Study Design and Sample}

Using resilience as the conceptual framework, the current study employed a crosssectional survey design to describe behavioral health risk and resilience across key sociodemographic factors among U.S. international students. Prospective participants consisted of foreign-born students who currently were foreign citizens and enrolled in a degree program (undergraduate or graduate) at a U.S. college/university with either an F-1 or J-1 visa. Data were collected using a multi-method survey approach that enabled respondents to either participate in an online survey or complete a paperbased questionnaire that was administered face to face. The study was approved by the Institutional Review Board of the primary researcher's affiliated university. 
Respondents for the online survey were recruited through social networking sites, including Facebook, where prospective participants were identified from the websites of international student clubs nationwide. To ensure a more representative sample and increase sample size, a maximum variation sampling method was used to select large universities with international student populations of 5,000 or more and smaller institutions with populations of less than 1,000. Information about U.S. international student populations was retrieved from the IIE website. The online survey was hosted on a web-based survey platform, Qualtrics, with biweekly follow-up reminders and survey information posted on each website. For the paper-based surveys, a convenience sampling method was used to recruit prospective participants from four large universities in a southern state. The principal investigator administered the survey face to face either with individual students or in small-group settings, whichever was most feasible. A total sample of 341 cases was collected (150 from the online survey and 191 from the paperbased survey) over a 5-month period of time (August 27, 2015 to January 17, 2016). However, due to missing data ( $>80 \%$ of items), 19 cases from the online survey were removed. The final sample included 322 participants: 131 cases from the online survey and 191 from the paper-based survey.

\section{Measurement}

The survey instrument consisted of 74 items in five major sections. Sociodemographic information was collected with 14 questions.

\section{Acculturative Stress}

A researcher-modified version of the Index of Life Stress (ILS; Yang \& Clum, 1995) scale was used to assess acculturative stress among participants. Used in previous research with Asian (Yang \& Clum, 1995) and with non-Asian international students in the US (e.g., Koyama \& Belli, 2011; Sullivan, 2010), the original, validated ILS contains 31 items comprising five subdomains of stressful life events (financial concerns, language difficulties, perceived discrimination, cultural adjustment, and academic pressure). Respondents are asked to select a number that best represents their personal experience living in the US, with response options range from 0 (never) to 3 (often). Item responses are summed yielding a total ILS scale score (Range $=0-93$ ), with higher scores indicating higher levels of acculturative stress. Based on the published results of previous validation studies (Yang \& Clum, 1995), and as recommended by Hair, Black, Babin, Anderson, and Tatham (2006), ILS items with factor loadings below 0.6 were eliminated in the current study. This yielded a shorter, 15-item version that demonstrated adequate internal consistency (at $\alpha=.82$ ), with total scores ranging from 0 to 45 . Examples of items retained in the modified ILS scale include: I am worried about my academic performance, my English makes it hard for me to understand lectures, and many opportunities are denied to me. 


\section{Resilience}

A researcher-modified version of the 31-item Resilience Scale for Adults (RSA) was used to measure respondents' protective and resilience factors. The RSA includes six subdomains (positive perception of self, positive perception of future, social competence, structured style, family cohesion, and social resources) and has been cross-culturally validated with various samples in different languages, including Norwegian (e.g., Friborg, Hjemdal, Rosenvinge, \& Martinussen, 2003), Persian (Jowkar, Friborg, \& Hjemdal, 2010), and French (Hjemdal et al., 2011). The RSA is measured on a 5-point Likert scale with response options ranging from 1 (strongly disagree) to 5 (strongly agree). Individual items are summed to obtain a total RSA score (Range = 31-165), with higher scores indicating higher levels of resilience (Hjemdal et al., 2011). Similar to the approach used with the ILS, published validation studies were reviewed to identify and eliminate RSA items with factor loadings below 0.6 (Hair et al., 2006), thereby yielding a 20-item RSA (Range $=20-100$ ) with adequate internal consistency reliability (at $\alpha=.80$ ). Examples of retained RSA items include: I enjoy being with other people and my family and I have a common understanding of what's important in life.

\section{Mental Health}

The 9-item Patient Health Questionnaire (PHQ-9; Kroenke, Spitzer \& William, 2001) was used to measure depression symptomology and the 7-item Generalized Anxiety Disorder (GAD-7) scale (Spitzer, Kroenke, Williams, \& Löwe, 2006) was used to measure symptoms of anxiety in the current study. The clinically validated PHQ-9 demonstrated satisfactory internal-consistency reliability ( $\alpha=.87$ ) and construct validity in Hahn's (2010) study, which sampled 648 international students from 74 countries. The PHQ-9 asks respondents to indicate how often they experienced certain problems during the prior 2-week period (e.g., feeling tired or having little energy, little interest or pleasure in doing things), with response options measured on a 4-point Likert scale ranging from 0 (not at all) to 3 (nearly every day). Total PHQ-9 scale scores of 5, 10, 15, and 20 indicate mild, moderate, moderately severe, and severe depression, respectively (Kroenke, Spitzer, \& Williams, 2001). A score of 10 or higher is considered a cut-off point, with a sensitivity of $88 \%$ for major depression (Kroenke et al., 2001). The GAD-7 asks respondents about the frequency of anxiety symptoms (e.g., trouble relaxing, worrying too much about different things) during the prior 2 weeks, with response options range from 0 (not at all) to 3 (nearly every day). GAD-7 scores of 5, 10, 15, and 20 indicate mild, moderate, moderately severe, and severe anxiety, respectively (Kroenke et al., 2001. A score of 10 or higher is a cut-point for clinically significant anxiety (Kroenke et al., 2006).

\section{Binge Drinking}

The alcohol-related questions of the Monitoring The Future Survey (MTF; Johnston, O'Malley, Miech, Bachman, \& Schulenberg, 2014) were used to assess the extent to which students in the current study engaged in binge-drinking experiences. The MTF defines binge drinking as consuming five or more drinks in a row, at least once, during the prior 2 -week period. The binge-drinking item was measured with response options ranging 
from 1 (none) to 6 (10 or more times); however, due to lack of variability, responses were dichotomized as no (0) and yes (1) for subsequent data analyses.

\section{Data Analysis}

A four-phased analysis was employed (using SPSS 20; IBM Corp,2011) to describe participants' characteristics and shed light on key differences across the four main latent variables (i.e., acculturative stress, resilience, mental health, and alcohol misuse). First, a normality test was conducted using Fisher's skewness and kurtosis coefficients to confirm that the data met the assumptions for parametric bivariate analyses. As seen in Table 1, the values for asymmetry and kurtosis of the subdomains for both acculturative stress and resilience fell within the acceptable range for demonstrating normal univariate distribution $(2$ and +2$)$, as did the values of skewness and kurtosis for depression and alcohol misuse (Brown, 1988). Next, a missing value analysis was conducted, revealing that all variables except for three had less than $2.2 \%$ of cases missing (i.e., previous educational experience, grade point average [GPA], and funding source). Thus, as recommended by Acuña and Rodrigues (2004), a multiple imputation method was employed.

Next, univariate statistics were used for descriptive purposes. Bivariate analyses were conducted in the final phase to examine differences in acculturative stress, resilience, anxiety, depression, and alcohol misuse across key sociodemographic characteristics. Pearson's product moment correlation coefficient was computed to examine interrelationships among variables measured at the interval level and the pointbiserial correlation coefficient was computed to estimate the degree of relationship between dichotomous and interval-level variables (Brown, 1988). Chi-square tests of independence were conducted to determine whether associations between binge drinking and key variables were independently distributed. Independent-samples $t$ tests and analyses of variance (ANOVAs) were computed to compare differences on measures of acculturative stress, resilience, and anxiety across sociodemographic variables measured at the nominal level (e.g., gender, marital status, and country of origin). Tukey's honestly significant difference (HSD) was used in conjunction with ANOVA for post hoc assessment.

\section{Results}

\section{Sociodemographic and Behavioral Health Characteristics}

As shown in Table 1, the sample was primarily male (55.6\%) and 25 years of age or less (69.3\%). The majority (61.2\%) was from either India (37.6\%) or China (23.6\%) and pursuing some type of graduate degree (71.4\%). Most student participants were unmarried (85.4\%) and not living with their families (81.7\%). As seen in Table 1, almost two thirds reported having a religious affiliation (62.7\%). Just over half of students had been in the US 1 year or less $(54.3 \%)$ and most had no prior experience as a student at another U.S. institution (68.9\%, See Table 1). Participants were most likely to report majoring in engineering (32.6\%), business $(28.0 \%)$, and social sciences (10.5\%) and well over half (55.5\%) reported GPAs between 3.5 and 4.0. As seen in Table 1, family funds were the primary source of financial support $(45.0 \%)$ reported by students. 
Table 1. Demographic characteristics of the sample $(\mathrm{N}=322)$.

\begin{tabular}{|c|c|c|c|}
\hline Variable & $\%(n)$ & Variable & $\%(n)$ \\
\hline Age & & Major & \\
\hline $18-25$ & $69.3(223)$ & Engineering & $32.6(105)$ \\
\hline $26-30$ & $22.7(73)$ & Business & $28.0(90)$ \\
\hline $31-35$ & $5.6(18)$ & Social sciences & $10.5(33)$ \\
\hline $36-40$ & $2.5(8)$ & Computer science & $9.6(31)$ \\
\hline Gender & & Other & $19.3(62)$ \\
\hline Female & $44.4(143)$ & Length of Stay & \\
\hline Male & $55.6(179)$ & Less than 6 months & $35.7(115)$ \\
\hline Religious affiliation & & 6 months to 1 year & $18.6(60)$ \\
\hline No & $37.3(120)$ & Up to 2 years & $16.5(53)$ \\
\hline Yes & $62.7(202)$ & More than 2 years & $29.2(94)$ \\
\hline Marital status & & GPA & \\
\hline Never married & $85.4(275)$ & $0-2.9$ & $44.5(143)$ \\
\hline Other & $18.2(59)$ & $3.5-4.0$ & $55.5(179)$ \\
\hline Family status & & \multicolumn{2}{|c|}{$\begin{array}{l}\text { Prior experience } \\
\text { studying at another institution in the US }\end{array}$} \\
\hline $\begin{array}{l}\text { Living without } \\
\text { my family }\end{array}$ & $81.7(263)$ & No & $68.9(222)$ \\
\hline Living with my family & $18.3(59)$ & Yes & $31.1(100)$ \\
\hline \multicolumn{4}{|l|}{ Country of origin } \\
\hline India & $37.6(121)$ & Current source of funding & \\
\hline China & $23.6(76)$ & GRA/GTA & $18.9(61)$ \\
\hline South Korea & $15.5(50)$ & Family funds & $45.0(145)$ \\
\hline Taiwan & $6.2(20)$ & Loans & $14.9(48)$ \\
\hline Other & $17.1(55)$ & Other & $21.2(68)$ \\
\hline \multicolumn{4}{|l|}{ Educational level } \\
\hline Bachelor's & $28.6(92)$ & & \\
\hline Master's & $55.6(179)$ & & \\
\hline Doctoral & $15.8(51)$ & & \\
\hline
\end{tabular}

Note. GPA = grade point average; GRA = graduate research assistantship; GTA =graduate teaching assistantship.

In terms of participants' behavioral health characteristics, the mean score on the measure of acculturative stress was 13.63; whereas the mean score assessing resilience was 103.97 (see Table 2). Mean scores on measures of depression and anxiety (at 7.58 and 6.79, respectively) indicated that the sample, overall, reported mild symptoms of both depression and anxiety. Cronbach's alpha for the PHQ-9 and GAD-7 was .91 and .92, respectively, indicating good internal consistency reliability for both. As seen in Table 2, less than one fourth of participants reported engaging in binge drinking (at 21.7\%). 
Table 2. Acculturative stress and behavioral health risk and resilience: Description of measures $(\mathrm{N}=$ 322).

\begin{tabular}{lccccc}
\hline & M or \%(n) & SD & Range & Skewness & Kurtosis \\
\hline Acculturative & 13.63 & 0.45 & $0-45$ & 0.844 & 1.085 \\
stress & & & & & \\
Resilience & 103.97 & 1.13 & $29-140$ & 0.684 & 0.450 \\
Depression & 7.58 & 0.35 & $0-27$ & 0.934 & 0.297 \\
Anxiety & 6.79 & 0.29 & $0-21$ & 0.787 & 0.166 \\
Binge drink- & $21.7(70)$ & - & - & - & - \\
ing & & & & & \\
\hline
\end{tabular}

\section{Correlations}

A correlation matrix was computed to examine interrelationships among key variables. As shown in Table 3, anxiety and depression were strongly and positively correlated (.797). Acculturative stress was positively and moderately correlated with both depression and anxiety (at .507 and .468, respectively). Table 3 shows that negative and relatively weak correlations emerged between resilience and the four variables measuring behavioral health risk; namely, depression (-.253), acculturative stress (-.234), anxiety (-.170), and binge drinking (-.147).

Table 3. Intercorrelations among acculturative stress and behavioral health risk and resilience $(\mathrm{N}=$ 322).

\begin{tabular}{|c|c|c|c|c|c|}
\hline & 1 & 2 & & & \\
\hline Acculturative stress & - & & & & \\
\hline Resilience & $-.234^{* * *}$ & - & & & \\
\hline Anxiety & $.468^{* * *}$ & $-.170^{* *}$ & - & & \\
\hline Depression & $.507^{* * *}$ & $-.253^{* * *}$ & $.797^{* * *}$ & - & \\
\hline Binge drinking & .032 & $-.147^{* *}$ & -.038 & .001 & - \\
\hline
\end{tabular}

\section{Acculturative Stress}

As shown in Table 4, international students between the ages of 36 and 40 and 31 and 35 reported significantly higher levels of acculturative stress $(M=21.10$ and 15.50, respectively) than those between the ages of 26 and 30 and 18 and $25(\mathrm{M}=14.47$ and 12.95, respectively). Thus, higher levels of acculturative stress distinguished older international students from younger ones. In terms of gender differences, females $(M=15.41)$ reported higher levels of acculturative stress than males $(\mathrm{M}=12.22$; see Table 4$)$. International students who were never married $(\mathrm{M}=13.04)$ reported lower levels of acculturative stress than those who were either married, divorced, separated, or widowed $(M=17.13)$; and students living with family members reported significantly higher levels of stress than 
those who were not $(M=17.24$ and 12.83 , respectively; see Table 4$)$. As seen in Table 4, international students whose length of stay in the US exceeded more than 2 years showed higher levels of acculturative stress than all students whose length of stay was less than that.

International students from India reported lower levels of acculturative stress than those from China, South Korea, Taiwan, and other countries. Further, those who came from countries other than the four major Asian countries had the highest levels of acculturative stress (see Table 4). Lower levels of acculturative stress, therefore, distinguished international students from India from those reporting all other nationalities. International students majoring in the social sciences $(\mathrm{M}=17.88)$ showed considerably higher levels of acculturative stress than did those majoring in computer science, engineering, business, and other areas of study $(\mathrm{M}=11.48,12.36,13.59$, and 12.83, respectively). Thus, higher levels of acculturative stress distinguished Social Science majors from all other majors. As seen in Table 4, undergraduate students reported higher levels of acculturative stress $(M=16.21)$ than graduate students at either the master's $(M=$ 12.12) or doctoral levels $(\mathrm{M}=14.31)$.

\section{Resilience}

Table 4 shows that students with a religious affiliation showed higher levels of resilience than students without such an affiliation ( $\mathrm{M}=106.65$ and 99.44, respectively). Students from countries other than the four major Asian countries $(M=107.64)$ and from India $(\mathrm{M}=106.83)$ demonstrated higher levels of resilience than those from South Korea $(\mathrm{M}=$ 103.06), China ( $M=99.04)$, and Taiwan $(M=97.50)$. In terms of educational level, graduate students at the doctoral $(\mathrm{M}=107.88)$ and master's levels $(\mathrm{M}=105.72)$ reported higher levels of resilience than undergraduate students ( $\mathrm{M}=98.38$; see Table 4$)$.

\section{Anxiety and Depression}

As seen in Table 4, female international students showed higher levels of anxiety than their male counterparts $(\mathrm{M}=7.57$ and 6.16 , respectively). Participants with a religious affiliation $(\mathrm{M}=7.38)$ showed more anxiety than did those without a religious affiliation $(M=5.79)$. In terms of academic performance, international students with GPAs below 3.0 reported higher levels of anxiety than those with GPAs of 3.0 and higher ( $M=14.50$ and 7.54, respectively; see Table 4). Students from countries other than the four major Asian nations and undergraduates demonstrated the highest mean anxiety scores $(\mathrm{M}=$ 8.45 and 8.04, respectively), approaching moderate levels $(\geq 10)$. In terms of depression, higher levels of depressive symptomology distinguished females $(M=8.43)$ from males $(M$ $=6.91)$ and also undergraduates $(M=9.32)$ from graduate students at the both the master's and doctoral levels ( $\mathrm{M}=7.30$ and 5.43, respectively). Among all student groups, mean depression scores approached clinical levels $(\geq 10)$ for both undergraduates $(\mathrm{M}=9.32)$ and students from countries other than Asian nations $(M=9.67$; see Table 4). 


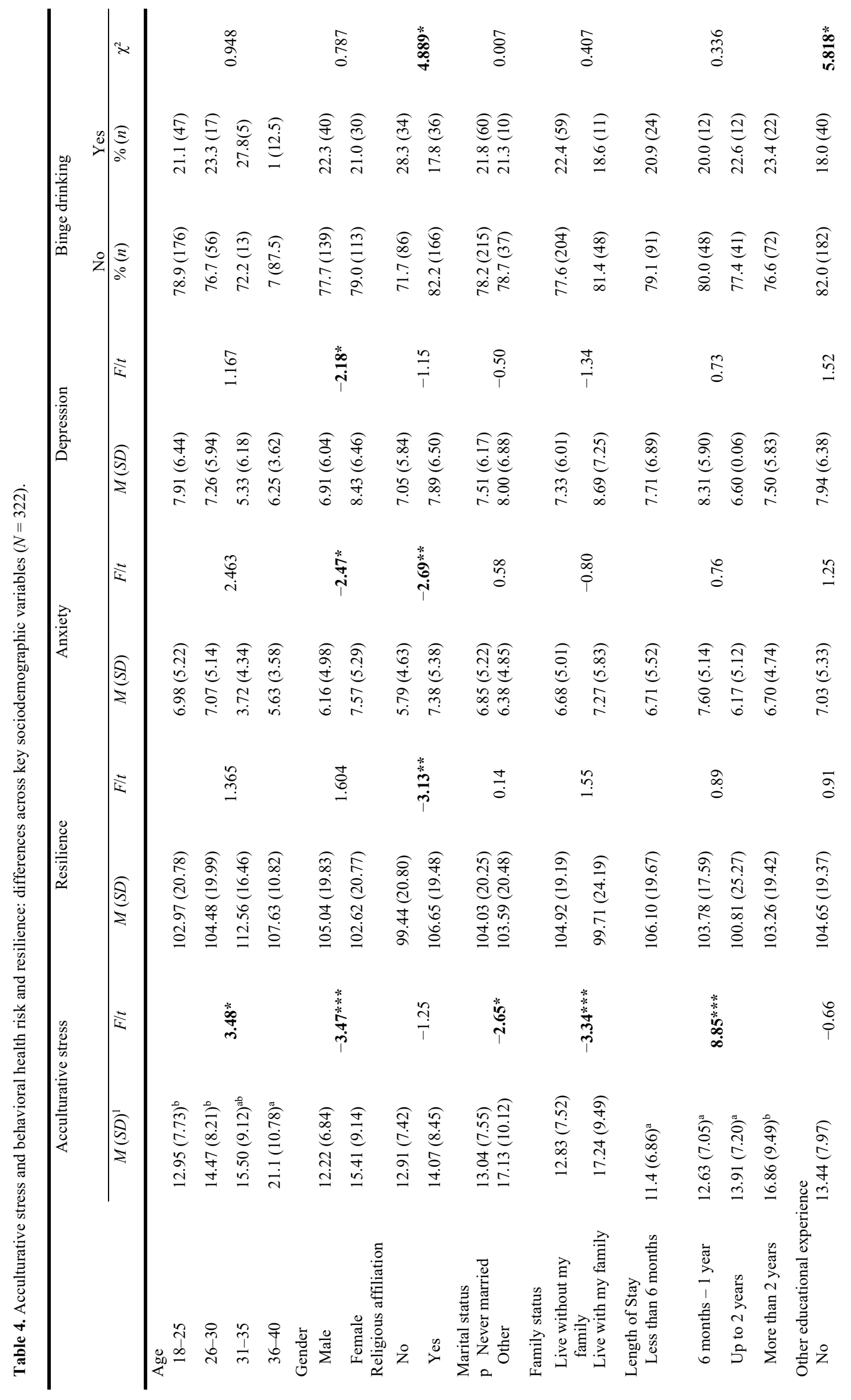




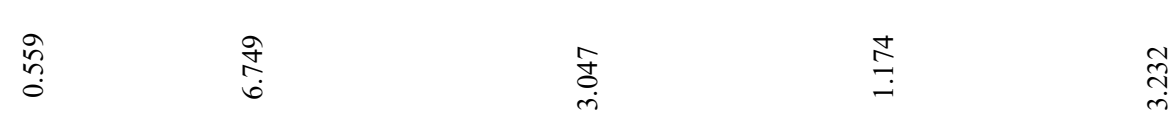

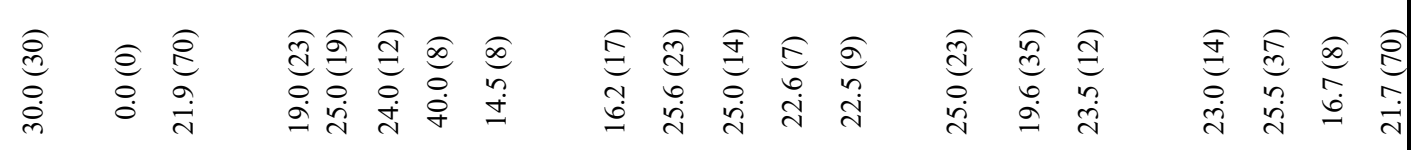

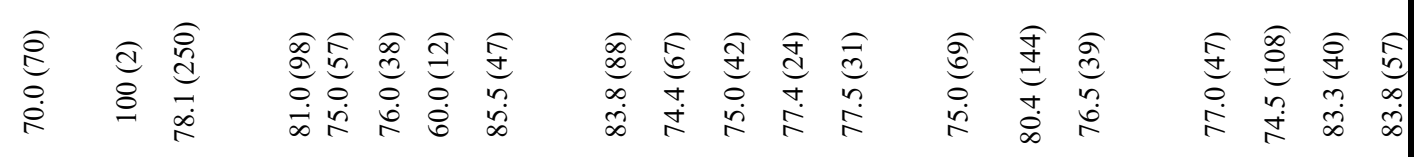
in
$\stackrel{8}{i}$
$\stackrel{8}{\circ}$
$\stackrel{n}{\stackrel{2}{*}}$

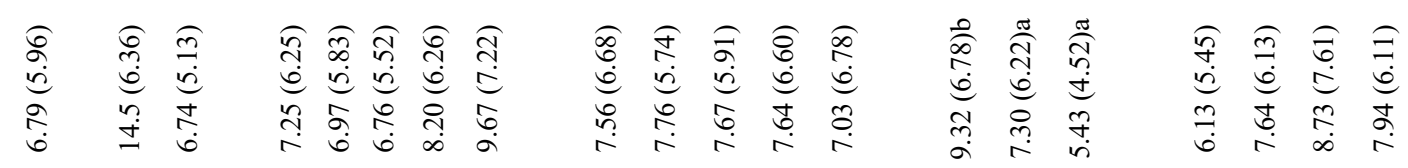<smiles>CCCC</smiles>

สี

$\frac{*}{4}$

ริ

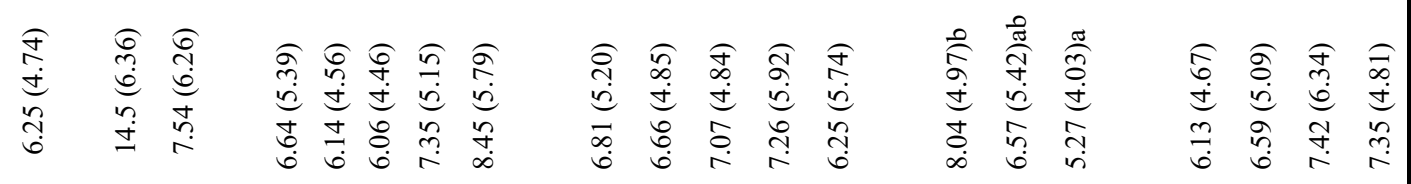

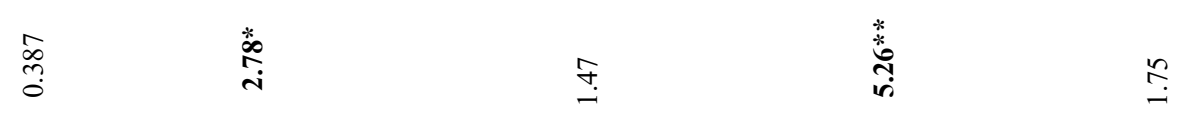

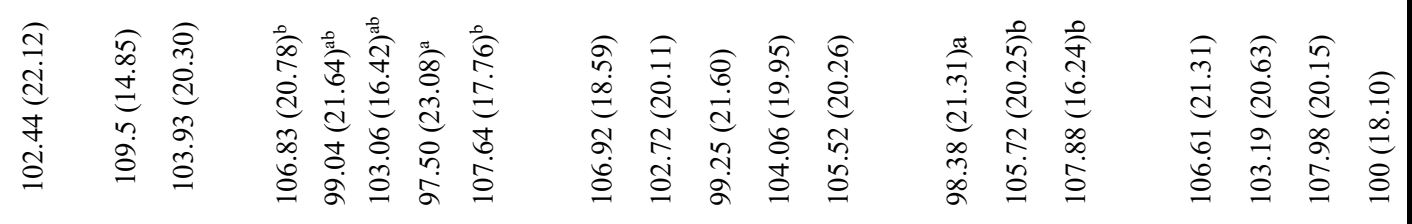
高

$\underset{\substack{* \\ 3}}{*}$

戠

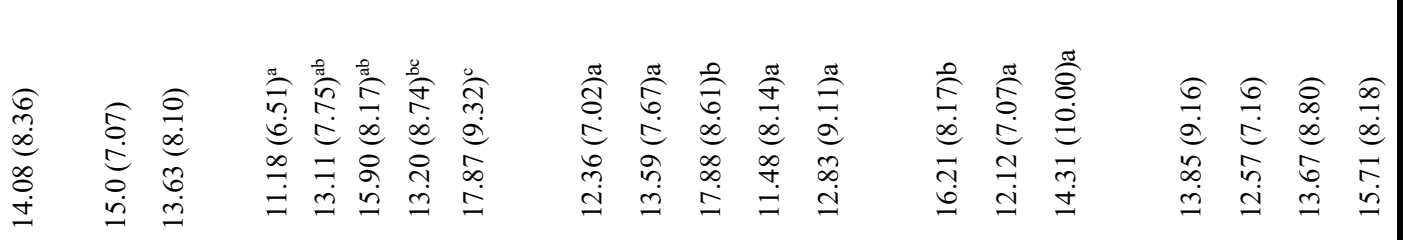

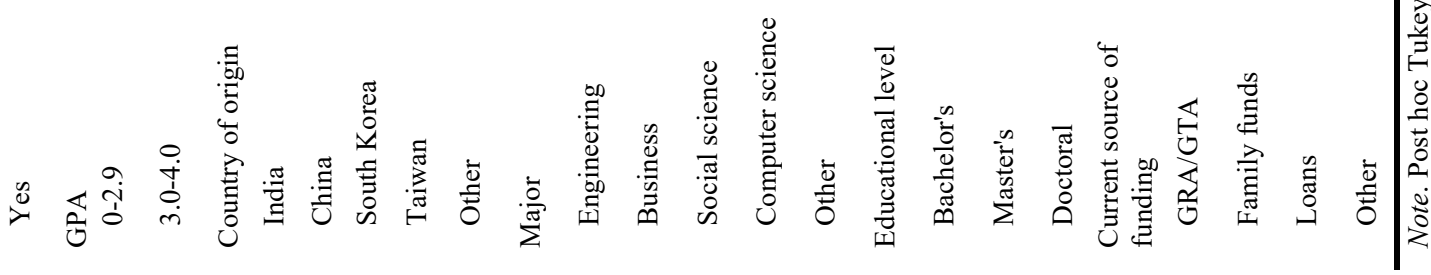




\section{Binge Drinking}

Table 4 shows that the proportion of international students who engaged in binge drinking was greater for students without any religious affiliation $(28.3 \%)$ than for those with a religious affiliation (17.8\%). International students with no prior educational experience in the US were less likely to have engaged in binge drinking than those with a prior educational history (at $18.0 \%$ and $30.0 \%$, respectively). As seen in Table 5, only one significant difference emerged across measures of anxiety, depression, resilience, and stress: Students who engaged in binge drinking showed lower levels of resilience $(\mathrm{M}=$ 98.31) than those who did not $(\mathrm{M}=105.54)$.

Table 5. Differences in Acculturative Stress and Behavioral Health Risk and Resilience by Binge Drinking Status $(\mathrm{N}=322)$

\begin{tabular}{|c|c|c|c|c|c|c|c|c|}
\hline \multirow[t]{2}{*}{$\begin{array}{c}\text { Binge } \\
\text { drinking }\end{array}$} & \multicolumn{2}{|c|}{$\begin{array}{c}\text { Acculturative } \\
\text { stress }\end{array}$} & \multicolumn{2}{|c|}{ Resilience } & \multicolumn{2}{|c|}{ Anxiety } & \multicolumn{2}{|c|}{ Depression } \\
\hline & $M(\mathrm{SD})$ & $t$ & $\begin{array}{l}M \\
(\mathrm{SD})\end{array}$ & $t$ & $\begin{array}{l}M \\
(\mathrm{SD})\end{array}$ & $t$ & $\mathrm{M}(\mathrm{SD})$ & $t$ \\
\hline No & $\begin{array}{l}13.5 \\
(7.99)\end{array}$ & -0.56 & $\begin{array}{l}105.54 \\
(18.82)\end{array}$ & $\begin{array}{l}2.66 \\
* *\end{array}$ & $\begin{array}{l}6.89 \\
(5.18)\end{array}$ & 0.68 & $\begin{array}{l}7.58 \\
(6.30)\end{array}$ & -0.01 \\
\hline Yes & $\begin{array}{l}14.13 \\
(8.45)\end{array}$ & & $\begin{array}{l}98.31 \\
(24.08)\end{array}$ & & $\begin{array}{l}6.41 \\
(5.11)\end{array}$ & & $\begin{array}{l}7.59 \\
(6.21)\end{array}$ & \\
\hline
\end{tabular}

${ }^{* *} \mathrm{p}<.01$

\section{Discussion}

The current study investigated sociodemographic differences across a host of behavioral health risk and resilience factors in the context of U.S. international academic mobility. Acculturative stress was associated with eight of the 12 sociodemographic characteristics under investigation, resilience and anxiety with three characteristics each, and depression and binge drinking with two each. Numerous significant intercorrelations emerged among the five primary outcomes of interest, with the strongest, not surprisingly, between anxiety and depression. In addition, both anxiety and depression showed moderate correlations with acculturative stress, demonstrating co-occurrence of behavioral health risk factors and acculturation-related stressors among participants in the current study.

\section{Acculturative Stress}

Consistent with prior research showing a positive relationship between age and acculturative stress (Lau, 2006), higher levels of stress emerged among older than 
younger students. Interestingly, undergraduate students showed higher stress levels than graduates. It is possible that graduate students, who were overrepresented in the sample, were slightly younger than undergraduates. On the other hand, the somewhat paradoxical findings suggest that adapting to a new academic and cultural environment may be especially challenging for older students with more entrenched traditional beliefs and practices, greater family responsibilities, or both. Also, the lower levels of stress among younger international students may reflect the fact that their length of residency at the time of the survey precluded the development of acculturation-related difficulties, an interpretation somewhat supported by the relatively high levels of acculturative stress observed among students whose length of stay exceeded 2 years. However, due to the cross-sectional design, it is unknown whether stress levels remain high and constant over time or fluctuate with the changing demands posed by the host country. Longitudinal research is needed to develop knowledge about the precise nature of this latter association among diverse U.S. international students.

In terms of gender, females in the current study demonstrated higher levels of acculturative stress than their male counterparts, a result that is somewhat at odds with previous research showing no such association (Eustace, 2007; Lau, 2006; Sullivan, 2010). Other studies have yielded mixed findings (e.g., Poyrazli et al., 2004), suggesting that it possibly may be culture-based, traditional gender roles, rather than gender, per se, that is associated with higher stress levels, an interpretation that is consistent with the considerably lower levels of acculturative stress reported by unmarried international students. This latter finding suggests the possible presence of a complex interplay of factors with regard to the role of the family for international students. Poyrazli and Kavanaugh (2006) suggested that the marital relationship has a stress-buffering effect; however, this latter study did not control for gender due to low power. The pressure of being responsible for the welfare and acculturation of spouses and other family members in an unfamiliar country may outweigh the presumed benefits of having family members nearby to provide needed social support. However, there are few studies about international students' experiences as partners and parents, leading Doyle, Loverridge, and Faamanatu-Eteuati (2016) to conclude that international students' accompanying family members are invisible in data collection systems, research, and policies pertinent to international education. A recent, small-scale, narrative analysis showed that international students with families do, in fact, have concerns about their children's adjustment as family members navigate numerous transitions in the host country (Loveridge, Doyle, \& Faamanatu-Eteuati, 2018). Additional research is needed to shed light on the influence of gender, marital status, children's experiences, and other family-related factors on the level of acculturative stress experienced by U.S. international students.

Levels of stress also varied according to students' nationality. In the current study, students from India had lower levels of acculturative stress than students from China, South Korea, Taiwan, and other countries, findings that are consistent with those of previous investigations conducted with Asian international students also showing higher levels of acculturative stress among Chinese students (e.g., Iwamoto \& Liu, 2010; Lau, 2006; Wei et., 2007). It is important to note that in the current study, international 
students in the social sciences (e.g., political science, sociology) demonstrated higher levels of acculturative stress than students in other areas of study, such as engineering, computer science, and other STEM fields. In addition to possible heightened demands for acculturation, international students in the social sciences receive less financial support than those in the STEM disciplines (SEVP, 2014). Students from countries other than India were overrepresented in the social science field, suggesting that the observed relationship between acculturative stress and academic major may be linked to students' nationality. Conversely, students from India in the current study were overrepresented in the STEM fields, which may contribute to a more positive collective cultural experience and enhance the availability of peer support, thereby mitigating the negative stress associated with the process of acculturation.

\section{Resilience}

Three demographic characteristics (i.e., religious affiliation, country of origin, and educational level) distinguished international students with higher levels of resilience from those with lower levels. International students reporting a religious affiliation showed higher levels of resilience than those reporting no such affiliation, a finding that is consistent with a large corpus of research undertaken with culturally diverse samples demonstrating a positive and robust association between religiosity and adaptive coping (see, e.g., Abu-Raiya \& Pargament, 2015; Javanmard, 2013; E. K. Lee \& Chan, 2009). Students from India in the current study showed higher levels of resilience than those from Asian countries, a finding that also may reflect the collective strength afforded by the notable overrepresentation of this particular subgroup in the overall international student sample.

Graduate students showed higher levels of resilience than undergraduates, a difference that might be explained by the older students' level of maturity. This latter interpretation, however, is somewhat at odds with previous research undertaken with international students in Korea showing no association between either age or education level and level of resilience (Cheung \& Yue, 2012; Yoo et al., 2014). Thus, given the substantive importance of relevant contextual factors (e.g., host country) when examining students' well-being and other outcomes, additional research investigating sociodemographic correlates of resilience among U.S. international students is warranted.

\section{Anxiety and Depression}

Consistent with previous research undertaken with U.S. international students (Khoshlessan \& Das, 2017), females in the current study showed higher levels of anxiety and depression than their male counterparts. Although the average GAD-7 and PHQ-9 scores were within the mild-to-moderate range, findings highlight the heightened vulnerability of female international students to mental health symptomology, a gender-specific issue that may merit attention when orienting international students to campus life. Prevention strategies could include screening mechanisms at university settings to gauge levels of mental health among international students. Students with lower GPAs $(<3.0)$ had higher levels of anxiety when compared to those with higher GPA ( $\geq 3.0)$, as would be expected, 
given universal expectations for high academic achievement from international students. Participants with some type of religious affiliation had higher anxiety levels than did those without a religious affiliation, a somewhat counterintuitive finding. However, it should be noted that the average level of anxiety was within the mild-to-moderate range. The result may be artefactual, given the sizable proportion of students, overall, reporting such an affiliation (at $62.7 \%$ ). It also is plausible that the relatively higher levels of anxiety experienced by some international students preceded a decision to affiliate with a particular religion. Thus, the relationship between anxiety symptoms and religious affiliation merits further longitudinal investigation.

As compared to graduate students, undergraduates showed higher levels of both mental health symptomology and acculturative stress. Previous research with international students, and those with low levels of English proficiency, in particular, has yielded similar findings regarding interrelationships among anxiety, depression, and stress (Yeh \& Inose, 2016; Ying \& Han, 2006). It is possible that undergraduate international students in the current study were more isolated and lonely than their graduate counterparts (Sherry et al., 2010), as well as had fewer coping resources, as evidenced by significantly lower levels of resilience. Taken together, the findings underscore those of previous studies demonstrating the heightened vulnerability of certain populations of international students to psychological distress (Hahn, 2011; Holguin, 2011; Sa et al., 2013).

\section{Binge Drinking}

International students with no prior U.S. educational experiences were less likely to engage in binge drinking than those who previously studied at other U.S. institutions, a finding that is consistent with the extant research emphasizing the protective benefits afforded by traditional cultural values (i.e., immigrant paradox, Kim \& Kim, 2014). It is possible that international students in the current study developed unhealthy patterns of alcohol use that paralleled those of their U.S. peers with whom they interacted socially (Yan \& FitzPatrick, 2016). Yet no differences in binge drinking emerged in the current study across the different lengths of stay, suggesting more complex interrelationships at play here. Overall, approximately one in five international students $(21.7 \%)$ engaged in binge drinking, a rate approximately half that of U.S. college students (at $40 \%$, National Institute on Alcohol Abuse and Alcoholism, 2015a), but concerning nonetheless, given the elevated risk posed by acculturative stress and its potential threat to international students' psychological wellbeing. Binge drinking is associated with other health-risk behaviors and poor academic achievement in U.S. college student populations (e.g., El Ansari, Stock, \& Mills, 2013) suggesting a need for culturally competent approaches for detecting alcohol misuse among U.S. international students.

International students without any religious affiliation were more likely to engage in binge drinking than those with a religious affiliation. Although the role of religion as a protective mechanism among international students has received some scholarly attention (e.g., Hsu et al., 2009), the stress-buffering benefits of religion and spirituality afforded to diverse immigrant populations has been widely discussed (e.g., Hodge, Cardenas, \& Montoya, 2001; Hsu et al., 2009). Among U.S. college students, religion is associated with reduced alcohol use (Galen \& Rogers, 2004; Thompson, 2017). Given 
the positive association that emerged between religious affiliation and resilience in the current study, the findings converge to tentatively suggest that religiosity may mitigate the stressors that render some international students vulnerable to binge-drinking behavior. However, these complex interrelationships warrant further multivariate testing.

\section{Implications}

Concerns about the adjustment and mental health of international students have emerged in the scholarly literature for approximately two decades (e.g., Bradley, 2000; Mori, 2000; Pan, Ng, Young, \& Caroline, 2017). Some of the observed difficulties can be attributed to a variety of adjustment issues, such as acculturative stress (Hans, Pistole, \& Caldwell; 2011), academic stressors (Liu, 2009), loss of social support (Cheung \& Yue, 2011), and racial discrimination (Yakunina, Weigold, \& McCarthy, 2011). However, these latter issues are linked to elevated levels of mental health symptomology, and, as evidenced by the current study, certain populations (e.g., females, undergraduates) are more vulnerable than others. Institutions that benefit from the presence of diverse international students should be proactive in identifying and intervening early on with those who struggle with anxiety, depression, substance misuse, and other emergent behavioral health issues. Previous research (e.g., Han et al., 2013; Kim \& Kim, 2014; Rahman \& Rollock, 2004), together with the findings of the current study, which underscored the co-occurrence of behavioral health risk factors and acculturation-related stressors among participants, have clear implications for campus-based interventions, including early identification through outreach and education and professional counseling. Further, study findings also provide implications for education policies pertaining to diverse needs and capacity of international students.

\section{Culturally Responsive Mental Health Services}

The importance of providing culturally sensitive mental health services to international students is universally emphasized by researchers. Use of translated materials to assess mental health concerns of students (Chalungsooth \& Schneller, 2011), providing culturally sensitive counseling services (Masuda et al., 2009), group assertiveness training (Tavakoli et al., 2009), and group cognitive behavioral intervention (as cited in Smith \& Khawaja, 2011) are some of the promising strategies that have shown to decrease mental health symptoms and increase post-migration growth among international students. Thus, although evaluation research is scant, there is some evidence indicating that psychosocial interventions are beneficial to international students who obtain professional counseling (Mori, 2000).

While international students are a diverse population group, there are some common adjustments issues they face across different sub-populations. These include acculturative stress, language barriers, discrimination, and so forth (Han et al., 2013). However, fewer international students utilize campus-based mental health services for professional counseling (Mori, 2000). Prior studies show that one third of 41 students dropped out of counseling after the intake session (Nilsson, Berkel, Flores, \& Lucas, 2004) and international students were rarely present for individual counseling (Yakunina, Weigold, 
\& McCarthy, 2011). Yakinina and colleagues (2011), therefore, recommended a group counseling approach might be a suitable intervention to address adjustment concerns and mental well-being of international students.

While literature has established that international students have lower utilization of mental health services (Mori, 2000), it should be noted that underutilization of services does not mean the problem does not exist. In fact, research suggests the cultural values of international students influence their willingness to seek professional counseling services. Students' perceptions of both self-stigma and public stigma toward counseling services influence help-seeking behavior. Effective and culturally responsive mental health services should therefore, seek to understand international students' attitudes toward counseling services as part of a comprehensive, culturally sensitive assessment process (Lee, Ditchman, Fong, Piper, \& Feigon, 2017). Between the cultural barriers and service environment, however, it is important to note that lack of cultural sensitivity is an obstacle to service utilization and delivery not just caused by language barriers, but a conflict between cultural outlook on life and values making provider-patient communication difficult (Maleku \& Aguirre, 2014).

Despite widespread acknowledgement and some evidence showing that psychosocial interventions may, in fact, be beneficial for some populations of international students, there is scant intervention research with this population. More intervention research studies are therefore needed to inform programs and interventions that can provide the most culturally responsive counseling services to international students.

\section{Culturally Responsive Outreach and Education}

Underutilization of mental health services among international students also highlights the need for outreach and education programs. Programs that use culturally responsive methods are particularly crucial as international students do not usually report to college counseling centers (Nilsson et al., 2004; Li et al., 2016). Early identification of potentially problematic adjustment issues may be therefore, accomplished via systematic outreach (Boone et al., 2011). Often times, international students may not be aware that they are experiencing heightened symptomology (Chalungstooth \& Schneller, 2011). Further, barriers to obtaining mental health services as indicated above include self-stigma about mental illness and stigma toward professional counseling services (Lee et al., 2017; Li et al., 2016; Masuda et al., 2009), which need creative and culturally responsive ways for outreach and education.

Outreach during orientation sessions can be the first step. International students can be provided with information about symptoms of both depression (Kroenke et al., 2001) and anxiety (Spitzer, Kroenke, Williams, \& Lowe, 2016), as well as research-based facts about recommended drinking limits for men and women (NIAAA, 2015b). Dissemination of translated materials in different languages to help international students express their mental health concerns can be very helpful (Chalungsooth \& Schneller, 2011). Outreach activities such as the "Let's Talk" program that provide informal consultation might also be very helpful (Boone et al., 2011) as these informal sessions can also be less stigmatizing. Prior research indicates that outreach programs can promote help-seeking behaviors among Asian students whose cultural values may render them unwilling to 
seek professional counseling services when needed (Lee et al., 2014). Given the needs of diverse international students, outreach and education programs can also be inclusive of international students who are parents and who may have concerns about their children's adjustment (Loveridge et al., 2018). It should also be noted that culturally responsive outreach and education can only be successful when international students themselves are engaged in the process. So, peer learning programs where some experienced international students are involved as mentors to new cohorts of international students can drive these campus-based initiatives.

\section{Cohesive Learning Environment}

Studies have indicated that effective advising of minority students is strongly associated with the advisor's ability to identify and address issues such as, acculturation, cultural myths and stereotypes, and value conflicts, often faced by diverse populations (Pan et al., 2017). Furthermore, issues of diversity and migration should be addressed more on college campuses, which will also educate students from mainstream groups to be more aware of issues faced by their international peers. Facilitation of such awareness will not only increase peer understanding and support between students from all backgrounds (Zhang, Larkin, \& Lucey, 2014), this will also increase awareness among university administrators. This awareness can link student interests with larger university systems that can then, support educational policies that help create a cohesive learning environment. Further this will also bolster the campus climate to be more equitable to all students (OkazawaRey, 2017).

\section{Research Implications}

While studies highlight that binge drinking is a public health challenge among college students in the US (Beck et al., 2008) and that binge drinking increases among international students as they try to conform to the drinking culture (Koyama \& Belli, 2011), literature that compare the rates of binge drinking among U.S. students and international students is sparse and fragmented. Future studies should explore these comparisons that would significantly inform programs and policies to bolster the campus climate for all students. Further, this will also contribute to the field of health risk behaviors among college students with pragmatic approaches. Similarly, U.S. college students are significantly facing many mental health challenges (Pace, Silk, Nazione, Fournier, \& Collins-Eaglin, 2018). While the causes of anxiety and depression among U.S. college students might be different from that of international students, studies that compare linkages and differences could decipher comprehensive mental health services that are equitable to all students based on individual needs. Similarly, since resilience is a strong protective factor against health risk behaviors (Chin, 2016), strengthening resilience levels of international students might have ripple effects on U.S. students and vice versa. More studies focusing on resilience are therefore needed to inform. 


\section{Limitations, Merits, and Conclusions}

As with all cross-sectional survey research, limitations are inherent and must be acknowledged, most notably in the areas of design, sampling, measurement, and analytic approach. A cross-sectional design does not permit inferences about the temporal order of conditions or events. Longitudinal designs are most appropriate for tracking changes in students' risk and resilience markers over time. The use of a convenience sampling method introduces possible biases that may affect the representativeness of the obtained sample. The majority of participants $(52.8 \%)$ for the paper-based survey were recruited from four large universities in one southern state and the use of social networking sites (for the online survey) precluded researchers from confirming the eligibility of prospective participants. Graduate students were disproportionately represented (71.4\%), as compared to national-level data (47.6\%; IIE, 2016), as were participants from India (at $37.6 \%$ and $14.0 \%$, respectively). Thus, the findings can be generalized only to U.S. international students sharing similar characteristics in comparable geographical and institutional contexts.

There also may have been issues with measurement reliability. Although the standardized tools used in the current study (i.e., ILS, RSA, PHQ-9, GAD-7) have been normed with diverse populations, it is possible that certain items were misunderstood by respondents with lower levels of English proficiency or unique cultural perspectives that resulted in differential perceptions of mainstream behaviors or symptoms (Furr \& Bacharach, 2008). In addition, the latter four instruments and the tool used to assess binge drinking were vulnerable to social desirability bias among student participants who may have been embarrassed, fearful of negative evaluation, or unnecessarily concerned about possible academic consequences. Finally, the modifications made to the ILS and RSA instruments may have compromised reliable measurement of these outcomes of interest.

Finally, the use of bivariate statistical approaches, appropriate for the exploratorydescriptive purpose of the current study, did not allow researchers to develop a more comprehensive understanding of factors affecting U.S. international students' well-being. Given the number of empirically relevant sociodemographic variables that emerged in the current study, a multivariate approach would enable researchers to determine the particular characteristics that best predict different risk and resilience outcomes among students.

Despite these latter limitations, the current study extends the knowledge base in several ways. It is one of the few studies to examine numerous sociodemographic and educational characteristics within a risk and resilience framework. The study was undertaken with a relatively sizeable and diverse sample of U.S. international students. Also, rather than focusing on static personality traits (e,g., Duru \& Poyrazli, 2007; Wei et al, 2007), the current study, which is concerned with more malleable markers of behavioral health, demonstrates how acculturative stress and symptoms of depression and anxiety, although conceptually related, differentially manifest for certain subpopulations of U.S. international students. For example, although older students showed higher levels of acculturative stress, younger students experienced higher levels of depressive symptomology. 
Findings of the current study highlight the diversity that characterizes U.S. international students, as well as underscores the potential relevance of a behavioral health risk and resiliency framework for understanding their experiences. As noted by Börjesson (2017), research describing the experiences of international students is a critical area of inquiry in migration studies, and the current study lays a solid foundation for additional investigations examining the predictive ability of key sociodemographic characteristics on international students' adaptation to the US and on their mental health and well-being. Results of the current study suggest the need to implement and test culturally responsive approaches that target distressed and at-risk international students. The research evidence, albeit scant, supports the use of targeted outreach and education, as well as psychosocial counseling with some populations of international students. Providing culturally responsive tailored interventions to each international student will be the first step in the right direction in creating cohesive learning environment conducive to all students.

\section{References}

Abu-Raiya, H. \& Pargament, K. I. (2015). Religious coping among diverse religions: Commonalities and divergences. Psychology of Religion and Spirituality, 7(1), 24-33.

Ansari, W. E., Stock, C., \& Mills, C. (2013). Is alcohol consumption associated with poor academic achievement in university students? International Journal of Prevention Medicine, 4(10), 1175-88.

Beck, K. H., Arria, A. M., Caldeira, K. M., Vincent, K. B., O'Grady, K. E., \& Wish, E. D. (2008). Social context of drinking and alcohol problems among college students. American Journal of Health Behavior, 32(4), 420-430.

Berry, J. W. (2005). Acculturation: Living successfully in the two cultures. International Journal of Intercultural Relations, 29, 697-712.

Börjesson, M. (2017). The global space of international students in 2010. Journal of Ethnic and Migration Studies, 43(8), 1256-1275.

Brown, J. D. (1988). Understanding research in second language learning: A teacher's guide to statistics and research design. Cambridge University Press, Cambridge.

Chai, P. P. M., Krägeloh, C. U., Shepherd, D., \& Billington, R. (2012). Stress and quality of life in international and domestic university students: Cultural differences in the use of religious coping. Mental Health, Religion \& Culture, 15(3), 265-277.

Chalungsooth, P. \& Schneller, G. R. (2011). Development of translation materials to assess international students' mental health concerns. Journal of Multicultural Counseling \& Development, 39(3), 180-189.

Cheung, C. \& Yue, X. (2013). Sustaining resilience through local connectedness among sojourn students. Social Indicators Research, 111(3), 785-800.

Cheung, C. K. \& Yue, X. D. (2012). Sojourn students' humor styles as buffers to achieve resilience. International Journal of Intercultural Relations, 36(3), 353-364.

Chin, F. P. (2016). A study of retention and achievement: Assessment of international students' resilience and coping strategies (Doctoral dissertation). Retrieved from ProQuest dissertations and theses. (Order, No., 10230223. 
Constantine, M. G., Ogazaki, S., \& Utsey, S. O. (2004). Self-concealment, social self-efficacy, acculturative stress, and depression in African. Asian, and Latin American international college students. American Journal of Orthopsychiatry, 74(3), 230-242.

Doyle, S., Loveridge, J., \& Faamanatu-Eteuati, N. (2016). Counting family: Making the family of international students visible in higher education policy and practice. Higher Education Policy, 29(2), 184-198.

Duru, E. \& Poyrazli, S. (2007). Personality dimensions, psychosocial-demographic variables, and English language competency in predicting level of acculturative stress among Turkish international students. International Journal of Stress Management, 14(1), 99-110.

E., A. \& Rodrigues, C. (2004). The treatment of missing values and its effect on classifier accuracy. pages 639-648, Chicago: Illinois. Institute of Technology.

Eustace, R. W. (2007). Factors influencing acculturative stress among international students. in the United States (Doctoral dissertation). Retrieved from ProQuest dissertations and theses.

Fergus, S. \& Zimmerman, M. A. (2005). Adolescent resilience: A framework for understanding healthy development in the face of risk. Public Health, 26, 399-419.

Friborg, O., Hjemdal, O., Rosenvinge, J. H., \& Martinussen, M. (2003). A new rating scale for adult resilience: What are the central protective resources behind healthy adjustment? International Journal of Methods in Psychiatric Research, 12, 65-76.

Furr, R. M. \& Bacharach, V. R. (2008). Psychometrics: An introduction. Thousand Oaks. Sage, CA.

Galen, L. W. \& Rogers, W. M. (2004). Religiosity, alcohol expectancies, drinking motives and their interaction in the prediction of drinking among college students. Journal of Studies on Alcohol, 65(4), 469-476.

Hahn, Z. L. (2010). Coping with acculturative stress and depression among international students: A cultural perspective (Doctoral dissertation). Retrieved from ProQuest dissertations and theses. (Order, No., 3447492.

Hair, J. F., Black, W. C., Babin, B. J., Anderson, R. E., \& Tatham, R. L. (2006). Multivariate data analysis. Pearson Prentice Hall, Upper Saddle River, NJ.

Han, X., Han, X., Luo, Q., Jacobs, S., \& Jean-Baptiste, M. (2013). Report of a mental health survey among Chinese international students at. Yale University. Journal of American College Health, 61(1), 1-8.

Hodder, R. K., Daly, J., Freund, M., Bowman, J., Hazell, T., \& Wiggers, J. (2011). A schoolbased resilience intervention to decrease tobacco, alcohol and marijuana use in high school students. BMC Public Health, 11(722), 1-10.

Hodge, D., Cardenas, P., \& Montoya, H. (2001). Substance use: Spirituality and religious participation as protective factors among rural youth. Social Work Research, 25(3), 153161.

Holguin, L. E. (2011). Substance use and social networks of international students (Doctoral dissertation). Retrieved from ProQuest dissertations and theses. (Order, No., 1495485.

Hsu, P. H., Krageloh, C. U., Shepherd, D., \& Billington, R. (2009). Religion/spirituality and quality of life of international tertiary students in New Zealand: An exploratory study. 
Mental Health, Religion \& Culture, 12(4), 385-399.

Iwamoto, D. K. \& Liu, W. M. (2010). The impact of racial identity, ethnic identity, Asian values, and race-related stress. on Asian Americans and Asian international college students' psychological well-being. Journal of Counseling Psychology, 57(1), 79-91.

Javanmard, G. H. (2013). Religious beliefs and resilience in academic students. Procedia: Social and Behavioral Sciences, 84, 744-748.

Johnson, N., Dinsmore, J. A., \& Hof, D. D. (2011). The relationship between college students' resilience level and type of alcohol use. International Journal of Psychology, $8,67-82$.

Johnston, L. D., O’Malley, P. M., Miech, R. A., Bachman, J. G., \& Schulenberg, J. E. (2014). Monitoring the Future national results on drug use: 1975-2013: Overview, key findings on adolescent drug use. Ann Arbor: Institute.

Jowkar, B., Friborg, O., \& Hjemdal, O. (2010). Cross-cultural validation of the Resilience Scale for Adults (RSA) in Iran. Scandinavian Journal of Psychology, 51, 418-425.

Kanaparthi, A. (2009). Relations between acculturation and alcohol use among international students (Doctoral dissertation). Retrieved from ProQuest dissertations and theses. (Order, No., 3394817.

Khoshlessan, R. \& Das, K. P. (2017). Analyzing international students' study anxiety in higher education. Journal of International Students, 7(2), 311-328.

Kim, M. \& Kim, K. (2014). A study of Chinese students' acculturative stress, depression influencing on drinking problem and social maladaptation: Moderating effect of ego resilience. Asian Journal of Child Welfare and Development, 12(2), 23-41.

Koyama, C. \& Belli, G. (2011). Alcohol use, acculturative stress, and drinking motivation among international community college students. Journal of Multicultural Counseling \& Development, 39(4), 229-240.

Kroenke, K., Spitzer, R. L., \& Williams, J. B. W. (2001). The PHQ-9 validity of a brief depression severity measure. Journal of General Internal Medicine, 16(9), 606-613.

Lau, J. S. (2006). Acculturative stress, collective coping, and psychological well-being of Chinese international students (Doctoral dissertation). Retrieved from ProQuest dissertations and theses. (Order, No., 3245416.

Laudet, A. B., Harris, K., Kimball, T., Winters, K. C., \& Moberg, D. P. (2015). Characteristics of students participating in collegiate recovery Programs: A national survey. Journal of Substance Abuse Treatment, 51, 38-46.

Lee, C. Y. \& Park, J. H. (2014). The effects of acculturative stress and resilience on depression of university students from North Korea. Family and Environment Research, 52(3), 313324.

Lee, E. K. \& Chan, K. (2009). Religious/spiritual and other adaptive coping strategies among Chinese American older immigrants. Journal of Gerontological Social Work, 52, 517-533.

Li, J., Marbley, A. F., Bradley, L. J., \& Lan, W. (2016). Attitudes toward seeking professional counseling services among Chinese international students: Acculturation, ethnic identity, and English proficiency. Journal of Multicultural Counseling \& Development, 44(1), 65-76. 
Loveridge, J., Doyle, S., \& Faamanatu-Eteuati, N. (2018). Journeys across educational and cultural borders: international postgraduate students with young children. British Journal of Sociology of Education, 39(3), 333-347.

Maleku, A. \& Aguirre, R. (2014). Culturally competent health care from the immigrant lens: a qualitative interpretive meta-synthesis. volume 29.

Pace, K., Silk, K., Nazione, S., Fournier, L., \& Collins-Eaglin, J. (2018). Promoting mental health help-seeking behavior among first-year college students. Health Communication, 33(2), 102-110.

Pan, J., Ng, P., Young, D. K., \& Caroline, S. (2017). Effectiveness of cognitive behavioral group intervention on acculturation. Research on Social Work Practice, 27(1), 68-79.

Poyrazli, S. \& Kavanaugh, P. R. (2006). Marital status, ethnicity, academic achievement, and adjustment strains: The case of graduate international students. College Student Journal, 40, 767-780.

Poyrazli, S., Kavanaugh, P. R., Baker, A., \& Al-Timimi, N. (2004). Social support and demographic correlates of acculturative stress in International students. Journal of College Counseling, 7, 73-82.

Sa, J. (2010). Binge drinking, drinking and driving, and cigarette smoking among Korean international. college students in the U.S. (Doctoral dissertation). Retrieved from ProQuest dissertations and theses. (Order No. 3432133.

Sa, J., Seo, D. C., Nelson, T. F., \& Lohrmann, D. K. (2013). Cigarette smoking among Korean international college students in the United States. Journal of American College Health, 61(8), 37-41.

Sherry, M., Thomas, P., \& Chui, W. H. (2010). International students: A vulnerable student population. The Journal of Higher Education, 60, 33-46.

Smith, R. A. \& Khawaja, N. G. (2011). A review of the acculturation experiences of international students. International Journal of Intercultural Relations, 35, 699-713.

Sondhi, G. \& Russell, K. (2017). Gendering international student migration: An Indian case-study. Journal of Ethnic and Migration Studies, 43(8), 1308-1324.

Spitzer, R. L., Kroenke, K., Williams, J. B. W., \& Lowe, B. (2016). A brief measure for assessing generalized anxiety disorder. Archives of Internal Medicine, 166, 1092-097.

Sullivan, C. (2010). Predictors of acculturative stress for international students in the United States (Doctoral dissertation). Retrieved from ProQuest dissertations and theses. (Order, No., 3432885.

Tavakoli, S., Lumley, M. A., Hijazi, A. M., Slavin-Spenny, O. M., \& Parris, G. P. (2009). Effects of assertiveness training and expressive writing on acculturative stress in international students: A randomized trial. Journal of Counseling Psychology, 56, 590-596.

Thompson, W. E. (2017). Social support, religious involvement and alcohol use among students at a conservative religious university. Behavioral Sciences, 7(2), 2-12.

Ungar, M. (2012). The social ecology of resilience. Springer, New York.

Wei, M., Heppner, P. P., Mallen, M. J., Ku, T., Liao, K. Y., \& Wu, T. (2007). Acculturative stress, perfectionism, years in the United States, and depression among Chinese international students. Journal of Counseling Psychology, 54(4), 385-394.

Yan, Z. \& FitzPatrick, K. (2016). Acculturation and health behaviors among international students: A qualitative approach. Nursing \& Health Sciences, 18(1), 58-63. 
Yang, B. \& Clum, G. A. (1995). Measures of life stress and social support specific to an Asian student population. Journal of Psychology and Behavioral Assessment, 17(1), 51-67.

Yeh, C. J. \& Inose, M. (2003). International students' reported English fluency, social support satisfaction, and social connectedness as predictors of acculturative stress. Counselling Psychology Quarterly, 29(3), 15-28.

Yeramaneni, S. \& Sharma, M. (2009). Predictors of alcohol use and binge drinking among Asian Indian students. American Journal of Health Studies, 24(2), 287-297.

Ying, Y. \& Han, M. (2006). The contribution of personality, acculturative stressors, and social affiliation to adjustment: A longitudinal study of Taiwanese students in the United States. International Journal of Intercultural Relations, 30(5), 623-635.

Yoo, M., Choi, S. Y., Kim, Y. M., Han, S., Yang, N., Kim, H., \& Son, Y. (2014). Acculturative stress, resilience, and depression among Chinese students in Korea. The Journal of Korean Academic Society of Nursing Education, 19(3), 320-329.

Zhang, Q., Larkin, C., \& Lucey, B. (2014). The economic impact of higher education institutions in Ireland: Evidence from disaggregated input output tables. Social Science Review Network.

\section{Author biography}

Youn Kyoung Kim , is an Assistant Professor in the School of Social Work at Louisiana State University at Baton Rouge, Louisiana. Her research interest focuses on resilience and behavioral health problems among youth.

Arati Maleku is an Assistant Professor in the College of Social Work at Ohio State University. Her research interests focus on the phenomenon of human migration, forced migration, social demography, and social determinants of immigrant health.

Catherine Lemieu is a Margaret Champagne Womack Professor in Addictive Disorders at Louisiana State University at Baton Rouge, Louisiana. Her research interests include substance use disorders and integrated health.

Xi Du is a doctoral student in the School of Social Work at Louisiana State University at Baton Rouge, Louisiana. Her research interests include immigrant children's positive development and family welfare.

Zibei Chen is a postdoc research fellow in the School of Social Work at University of Michigan at Ann Arbor, Michigan. Her interests include financial behaviors, financial literacy, and access to financial services among low-income households. 